\title{
Alleviation of hepatic oxidative stress by Chinese herbal medicine Yin-Chen-Hao-Tang in obese mice with steatosis
}

\author{
TZUNG-YAN LEE ${ }^{1}$, HEN-HONG CHANG ${ }^{1,2}$, WEN-CHAI LO ${ }^{1}$ and HAN-CHIEH LIN ${ }^{3}$ \\ ${ }^{1}$ Graduate Institute of Traditional Chinese Medicine, Chang Gung University; ${ }^{2}$ Center for Traditional Chinese Medicine, \\ Chang Gung Memorial Hospital, Taoyuan; ${ }^{3}$ Division of Gastroenterology, Department of Medicine, \\ Taipei Veterans General Hospital, Taipei, Taiwan, R.O.C.
}

Received January 18, 2010; Accepted March 1, 2010

DOI: 10.3892/ijmm_00000412

\begin{abstract}
Obesity is associated with a complex systemic inflammatory state that has been implicated in the development of hepatic steatosis. This study was to test the efficacy of Yin-Chen-Hao-Tang (YCHT), an agent that improves hepatic triglyceride metabolism in its ability to modulate pathways implicated in hepatic steatosis. Mice were fed with high-fat diet for fifteen weeks. The therapeutic mechanism of YCHT likely enhanced adiponectin and endothelial progenitor cells, and up-regulation of peroxisome proliferator-activated receptor $\gamma$ might be responsible for fatty liver diseases. In addition, YCHT anti-oxidative stress effect might be associated with inhibition of hepatic free fatty acid concentrations and elevation of the glutathione levels in hepatic tissues. Furthermore, YCHT action mechanisms might promote senescence marker protein-30 metabolism that increase resistance to hepatic oxidative stress. These findings suggest a novel therapeutic approach for fatty liver progression in obesity mice.
\end{abstract}

\section{Introduction}

Obesity appears to augment the incidence of cardiovascular events and is associated with major risk factors for atherosclerosis, diabetes, metabolic syndrome (1) and coexisting pathological conditions frequently associated with nonalcoholic fatty liver disease (NAFLD) (2). Fat intake has been shown to induce inflammation, potentially through increases in oxidative stress (3). NAFLD ranges from simple triglyceride accumulation in the cytoplasm of hepatocytes (steatosis) to inflammatory and hepatocellular injury (nonalcoholic steatohepatitis) that can eventually lead to fibrosis and cirrhosis $(4,5)$. Hepatic steatosis occurs in conditions of

Correspondence to: Dr Tzung-Yan Lee, Graduate Institute of Traditional Chinese Medicine, Chang Gung University, No. 259, Wen-Hwa 1st Road, Kwei-Shan Tao-Yuan 333, Taoyuan, Taiwan, R.O.C.

E-mail: joyamen@mail.cgu.edu.tw

Key words: oxidative stress, herbal medicine, steatosis increased lipogenesis, defective mitochondrial/peroxisomal ß-oxidation, and/or a diminished ability of the liver to export lipids. Furthermore, the maintenance of a low oxidative stress status in the liver constitutes an additional requisite for optimum lipid homeostasis. Progression of hepatic steatosis into steatohepatitis can be promoted by oxidative stress, which is substantially exacerbated in the latter stages of NAFLD. In addition, research suggests that reduced adiponectin levels can occur due to lifestyle changes that cause obesity, such as a high-fat diet $(6,7)$. According to these observations, reduced adiponectin levels may play a causal role in the development of insulin resistance, metabolic syndrome, type 2 diabetes, and atherosclerosis. Peroxisome proliferator-activated receptor $\gamma$ (PPAR $\gamma$ ) agonists, used clinically to improve insulin sensitivity, also reduce liver steatosis and lipemia in rodent models (8) and more modestly in humans (9). The antisteatotic action of PPAR $\gamma$ agonism is thought to derive largely from PPAR $\gamma$ action in adipose tissue and reduced portal fatty acid delivery to the liver $(8,9)$ in addition to increased adiponectin secretion (10). Thus, efforts to enhance PPAR $\gamma$ activity might improve fatty liver disease.

Yin-Chen-Hao-Tang (YCHT) is recognized as a hepatoprotective agent for various types of liver diseases. YCHT consists of the following three medicinal herbs: Artemisia capillaries Thunb, Gardenia jasminoides Ellis, and Rheum officinale Baill. This decoction has long been used in Taiwan and China as an anti-inflammatory, antipyretic, and choleretic agent for liver disorders and jaundice. A variety of biological effects caused by YCHT have been described including potent effects on hepatic fibrosis $(11,12)$ and hepatic apoptosis both in vitro $(13)$ and in vivo $(14,15)$. In addition, upregulation of ApoA-I in bile duct ligation rats after YCHT administration indicated enhanced triglyceride metabolism, which may link ApoA-I deficiency to the pathophysiology of liver fibrosis (15).

The primary objective of this study was to identify potential mechanisms of YCHT for fatty liver disease, one of the most clinically significant complications of obesity-related syndrome. Our second objective was to identify the therapeutic potential of YCHT in obese mice. To investigate the underlying mechanisms of obesity-induced fatty liver, we used matrix-assisted laser desorption/ionization (MALDI)-based proteomics approach to identify candidate molecules that link high fat consumption to the pathogenesis of hepatic steatosis. 
We show that high fat feeding was associated with significant alterations in the expression of hepatic proteins in multiple pathways. These data provide new insight into the molecular basis that links fat-induced metabolic shift to aberrant hepatic metabolism in the pathogenesis of dyslipidemia and steatosis. In addition, YCHT may be useful for treatment of liver steatosis because of its possible anti-oxidant properties, and the therapeutic effects of YCHT on liver diseases might be associated with its lipid biosynthesis regulation.

\section{Materials and methods}

Obesity induction and YCHT treatments. Hamsters (6 weeks old) were obtained from the Taiwan NSC Animal Center and free access to water and one of two diets was supplied ad libitum for 15 weeks. Regular diet (Laboratory Autoclavable Rodent Diet 5010, Purina Mills Inc., Richmond, IN) had a caloric content of $3.4 \mathrm{kcal} / \mathrm{g}$ (calories from fat $13 \%$, protein $28 \%$, carbohydrates $59 \%$ ); the high fat diet (Mouse Diet High Carbohydrate High Fat F3282, BioServ, Frenchtown, $\mathrm{NJ}$ ) was $5.3 \mathrm{kcal} / \mathrm{g}$ (calories from fat $59 \%$, protein $16 \%$, carbohydrate $24 \%$ ). The mice were maintained in accordance with the Guidelines for the Care and Use of Laboratory Animals of Chang Gung University. YCHT was prepared as described in our previous studies (14). YCHT was dissolved in distilled water and administered daily $(250 \mathrm{mg} / \mathrm{kg}$ p.o.) from week 12 to 15 after feeding on a high fat diet. Liquid chromatography/electrospray ionization time-of-flight mass spectrometry (LC/ESI-TOFMS) was used to clarify the chemical composition of YCHT.

Histopathology assay, cytokine array assay and 2-D gel electrophoresis. The liver tissue was fixed in $10 \%$ formalin and then embedded in paraffin, cut into 5- $\mu \mathrm{m}$ thick sections, stained with $H \& E$, and examined under light microscopy by an experienced pathologist. A serum cytokine array was carried out with a RayBio ${ }^{\mathrm{TM}}$ cytokine array kit (RayBiotech, Inc., Norcross, GA) according to the manufacturer's protocol. Serum adiponectin, TNF- $\alpha$ and MCP- 1 were measured by commercial ELISA kits (R\&D Systems, Minneapolis, MN). Serum triglycerides were determined by spectrophotometry with the use of a RANSOD kit (RANDOX Laboratories Ltd., UK). A free fatty acid quantification kit was purchased from BioVision Research, CA, USA. Changes in GSH/GSSG were determined by HPLC (16). Two-dimensional-PAGE was performed according to our previous study to detect various proteosomal changes (15).

Flow cytometry. Mobilization of endothelial progenitor cells (EPCs) into the circulation was studied using flow cytometry. Cells isolated from mice were incubated with various Abs (BD Biosciences). Isotype-matched mouse immunoglobulins served as controls. One million viable cells were scanned, and lymphocytes were gated and analyzed using flow cytometer (BD Biosciences). The data were analyzed using Flow software.

Western blotting. Liver tissue was lysed with distilled water containing protease inhibitors (BD Pharmingen) and a Bio-Rad rapid Coomassie kit was used to determine the total protein concentration. Sixty micrograms of protein was run on a $10 \%$ SDS-PAGE gel followed by Western blotting with various mouse or rabbit monoclonal antibodies PPAR $\gamma$, SMP30, and Histone H1 from Santa Cruz or anti-actin antibody (Chemicon, USA). Chemiluminescence (ECL; Amersham, Piscataway, NJ) in conjunction with video densitometry was used to quantify protein expression.

Reverse transcription-polymerase chain reaction ( $R T-P C R)$. Freshly collected tissues were flash frozen on dry ice, processed shortly thereafter, and homogenized in $1 \mathrm{ml}$ of TRIzol solution (BRL, Gaithersburg, MD) for extraction according to the manufacturer's instructions. cDNA synthesis was achieved with SuperScript III reverse transcriptase (Invitrogen, Carlsbad, CA). Commercially available PCR primers were purchased from Purigo Biotech, Inc. (Taipei, Taiwan, R.O.C.) and contained the following sequences: peroxisome proliferators-activated receptor $\gamma(\operatorname{PPAR} \gamma)$ : forward 5' cat gct tgt gaa gga tgc aag $3^{\prime}$, reverse $5^{\prime}$ ttc tga aac cga cag tac tga cat $3^{\prime}$; tumor necrosis factor- $\alpha$ (TNF- $\left.\alpha\right)$ : forward 5' agc aca gaa agc atg atc cg 3', reverse 5' gta tga agt ggc aaa tcg gc 3'; fatty acid synthase (FAS): forward 5' tca cca ctg tgg get ctg cag aga agc gag $3^{\prime}$, reverse 5' tgt cat tgg cet cct caa aaa ggg cgt cca 3'; carnitine palmitoyl transferase-1 (CPT-1): forward 5' gga cag aga ctg tgc gtt cet 3', reverse 5' gcg ata tcc aac agt get tga 3'; GAPDH: forward 5' ccc ttc att gac ctc aac tac att gg 3', reverse 5' cat ggt ggt gaa gac gcc ag 3'.

Statistics. The results are expressed as mean \pm SEM. Statistical analyses were performed using one-way analysis of variance (ANOVA). Significance was set at $\mathrm{P}<0.05$.

\section{Results}

The aqueous extract of YCHT was analyzed by Agilent 6510 Q-TOF. The separated compounds were identified with pure standards, or characterized by analyzing their mass spectra recorded in both negative and positive ion polarity modes. Further structural information was obtained from in-source fragmentation. All the compounds identified in YCHT could be detected in one of the individual herbs, and no significant new compound was observed after the preparation process. Although we did discover a few minor peaks in the profile of YCHT that were only present in trace amounts and could not be identified. On the other hand, most of the abundant compounds in the individual herbs were detected in YCHT as well (Fig. 1).

Histological examination of liver sections from obese mice showed a significant change in the profile of foaming and ballon hepatocyte deposition compared to mice that received YCHT treatment. The obese mice treated with YCHT (250 mg/ $\mathrm{kg}$ ) had a significant reduction in granulocyte neutrophil accumulation (Fig. 2).

In order to determine the effect YCHT may have on cytokine expression, cytokine arrays were scanned (Fig. 3A). YCHT has shown a significant down-regulation of high fat diet-induced TNF- $\alpha$ and MCP-1 (Fig. 3C) levels. In addition, the amount of adiponectin (Fig. 3D) levels, positively correlated with EPC mobilization, increased with YCHT treatment (Fig. 3E). 


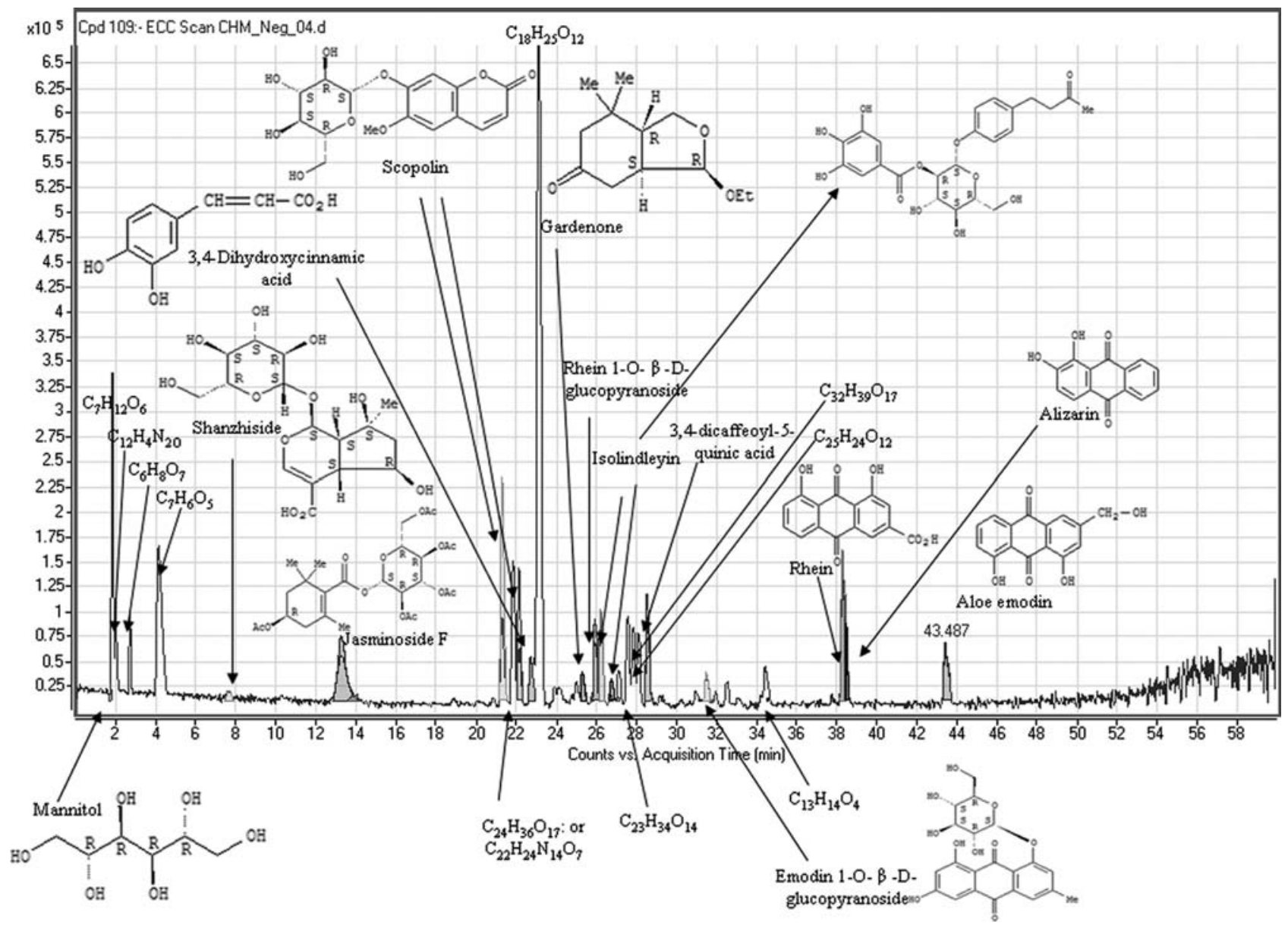

Figure 1. Liquid chromatography-time-of-flight mass spectrometry (LC-TOF/MS) was used to clarify the chemical composition of YCHT.

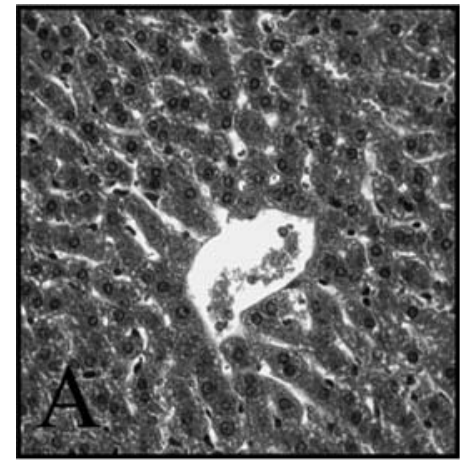

ND

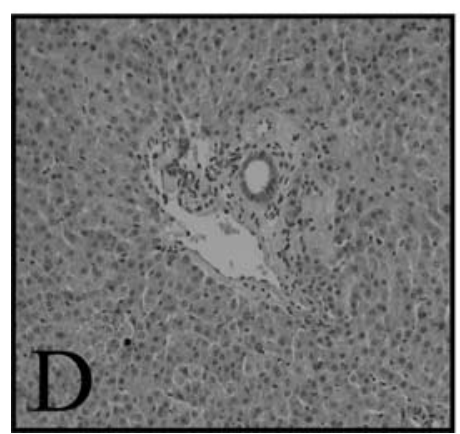

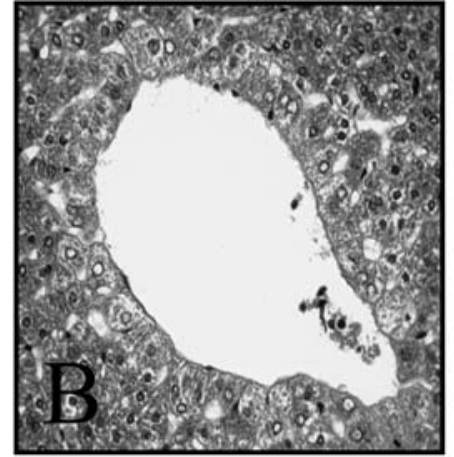

HFD

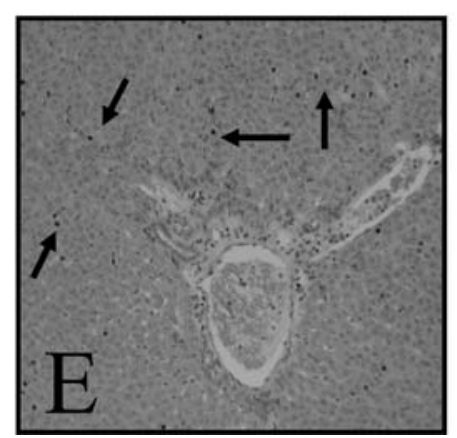

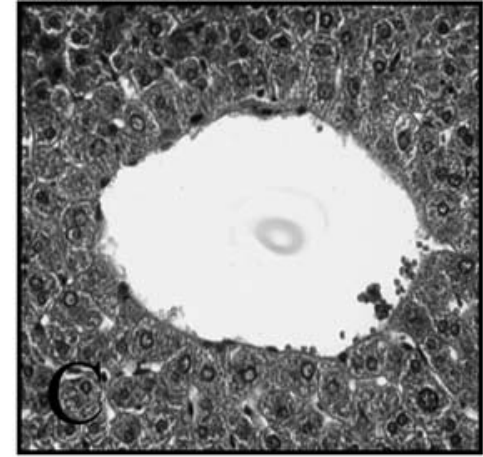

$\mathrm{HFD}+\mathrm{YCHT}$

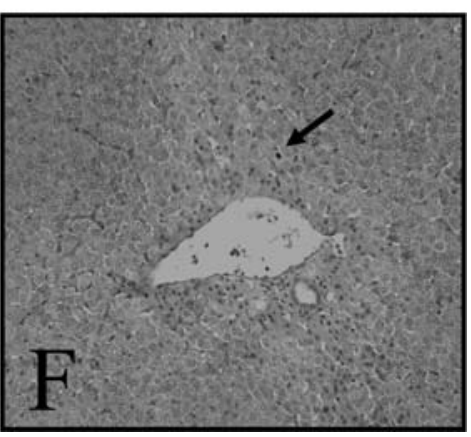

Figure 2. H\&E liver sections showing protective effect of YCHT treatment on high-fat diet-induced obese mice. There are significant balloon liver cells adjacent to the central vein (B) and granulocyte neutrophils adjacent to the portal triad (E) (arrowhead denotes inflammatory foci). In contrast, with YCHT treatment show cell patterns that are more ordered and show no signs of balloon hepatocytes. Normal diet (A, D), HFD (B, E) and YCHT treatment (C, F). Original magnification $\mathrm{x} 100$. 
(A)

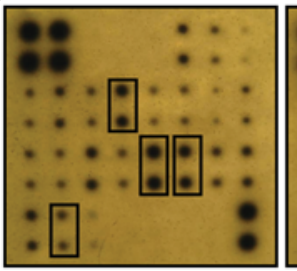

ND

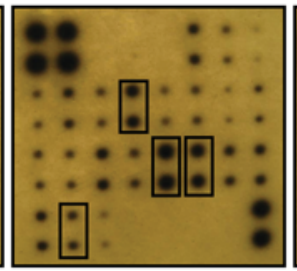

HFD

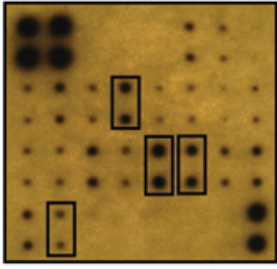

HFD+YCHT

(B)

\begin{tabular}{|c|c|c|c|c|c|c|c|c|}
\hline & A & B & c & D & E & F & G & H \\
\hline 1 & POS & POS & NEG & NEG & Blank & bFGF & $\mathrm{CD} 40$ & Eotaxin \\
\hline 2 & POS & POS & NEG & NEG & Blank & bFGF & $\mathrm{CD} 40$ & Eotaxin \\
\hline 3 & GCSF & GM-CSF & $\mathbb{I N F} \gamma$ & IL-1 $\alpha$ & IL-1 $\beta$ & IL-2 & IL-3 & II- 4 \\
\hline 4 & GCSF & GM-CSF & $\mathrm{INF} \gamma$ & LL- $1 \alpha$ & IL-1 $\beta$ & ㅍ-2 & IL-3 & II- 4 \\
\hline 5 & IL-5 & ㅍ-6 & IL-13 & L-Selectin & MCP1 & M-CSF & MIP-3 $3 \alpha$ & P-Selectin \\
\hline 6 & IL-5 & 프-6 & IL-13 & L-Selectin & MCP1 & M-CSF & $\mathrm{MIP}-3 \alpha$ & P-Selectin \\
\hline 7 & RANTES & TNF $-\alpha$ & VEGF & Blank & Blank & Blank & Blank & POS \\
\hline 8 & RANTES & TNF- $\alpha$ & VEGF & Blank & Blank & Blank & Blank & POS \\
\hline
\end{tabular}

(E)

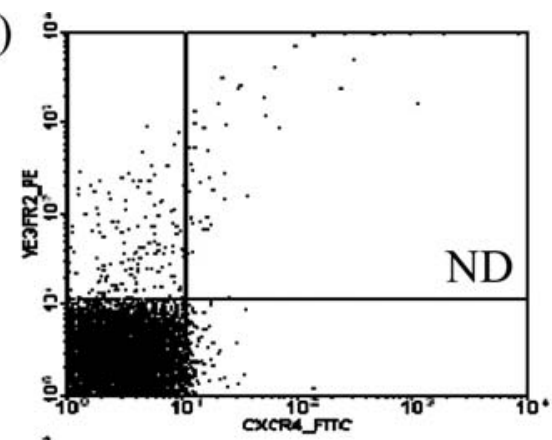

(C)

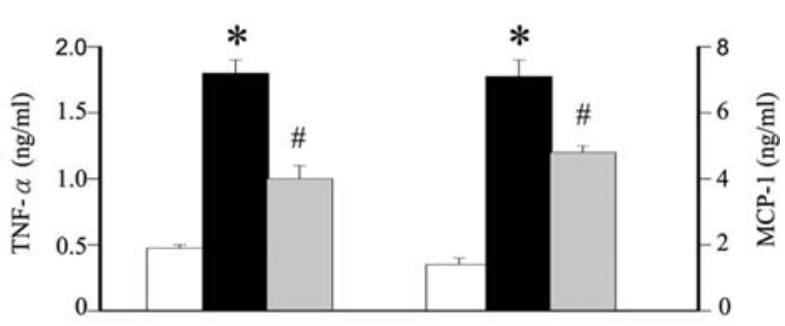

(D)

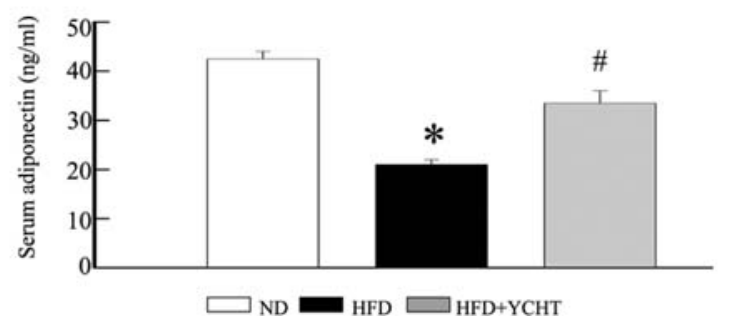

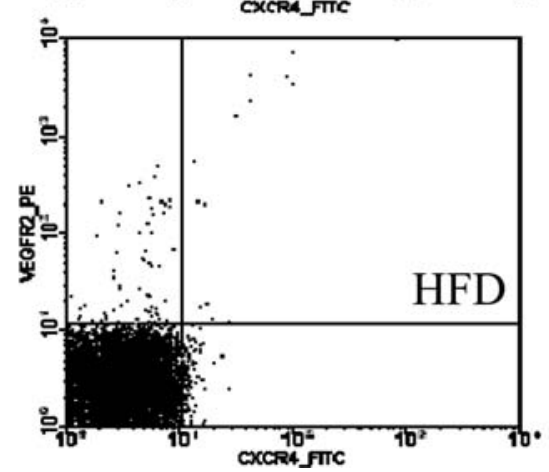
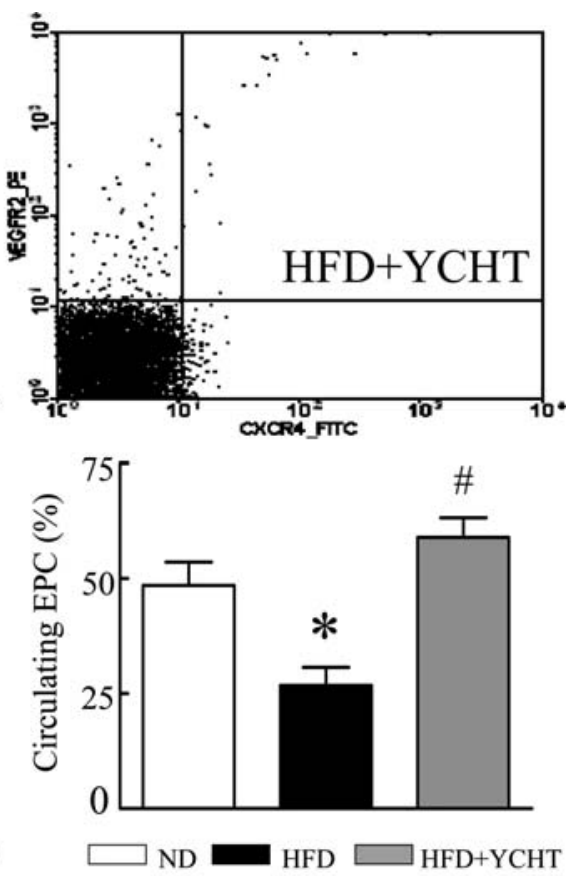

Figure 3. Array patterns of cytokine expression from the serum of obese mice with or without YCHT treatment (A). Cytokine array layout (B). Serum TNF- $\alpha$, MCP-1 (C) and adiponectin (D) levels were measured by ELISA. The effect of YCHT on endothelial progenitor cells (EPCs) in obese mice by fluorescentactivated cell sorter analysis. The levels of circulating EPCs were defined by CXCR4/VEGFR2 cells (E). Values are expressed as mean \pm SEM. ${ }^{*}<0.05$ compared to the normal diet; ${ }^{~} \mathrm{P}<0.05$ compared to the high fat diet. Blot shown is representative of three similar experiments.

YCHT decreased the expression of the fat oxidation genes CPT1 and FAS (Fig. 4). Changes in the expression of FAS and CPT-1 were accompanied by a detectable increase in PPAR $\gamma$ mRNA expression. Therefore, treatment of obese mice with YCHT also caused a marked reduction in the level of TNF- $\alpha$ expression. Compared to the untreated group, YCHT affected the total GSH (Fig. 4C and D), hepatic free fatty acid (Fig. 4E) and serum TG concentrations (Fig. 4F). These changes are known to be associated with increased PPAR $\gamma$ levels (Fig. 4A and B). Using 2-DE, the differential expression of molecules retrieved from liver of experimental animals were analyzed, respectively. Ten protein spots in liver tissue of the animals were analyzed (Table I). Protein spots were retrieved from the 2-DE gel and processed using in gel enzymatic digestion and MALDI-TOF, which gave rise to the final identification. From the identified proteins in the liver, 27 showed a significant effect in obesity mice and 33 were significantly modulation after YCHT treatment in obese mice. Among the variation proteins, enzymes related to the glucose metabolism (glucose regulated protein 78 , fructose-1,6-bisphosphatase 1) and oxidative stress-related proteins (electron transfer flavoprotein, heat shock protein, peroxiredoxin 6, glutathione-S-transferase, selenium binding protein 1) were affected. Additionally, aldehyde dehydrogenase 2 and regucalcin (SMP30) also shown significantly change. It should be pointed out these changes in protein levels were statistically significant or reached the usual threshold of 2-fold up- or down-regulation in YCHT treatment groups. From the identified proteins in the liver, nine candidates were significantly reduced or induced in 
(A)

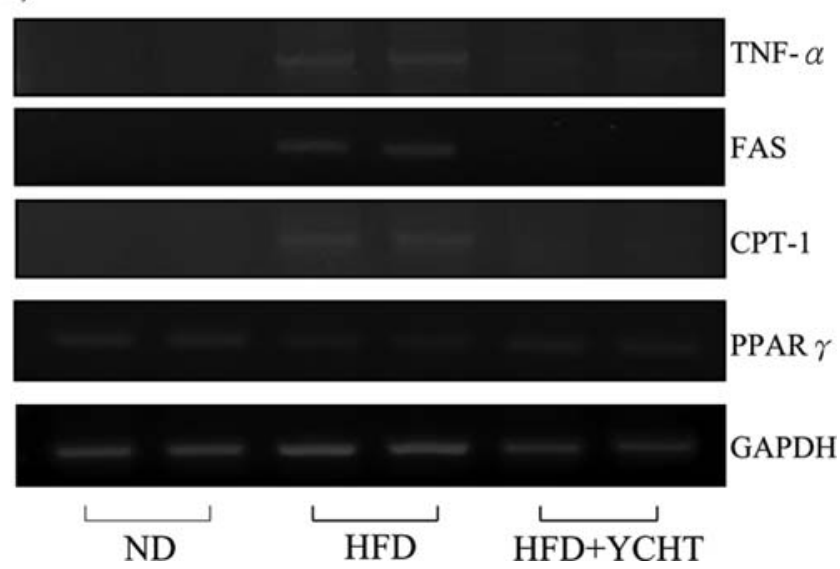

(B)
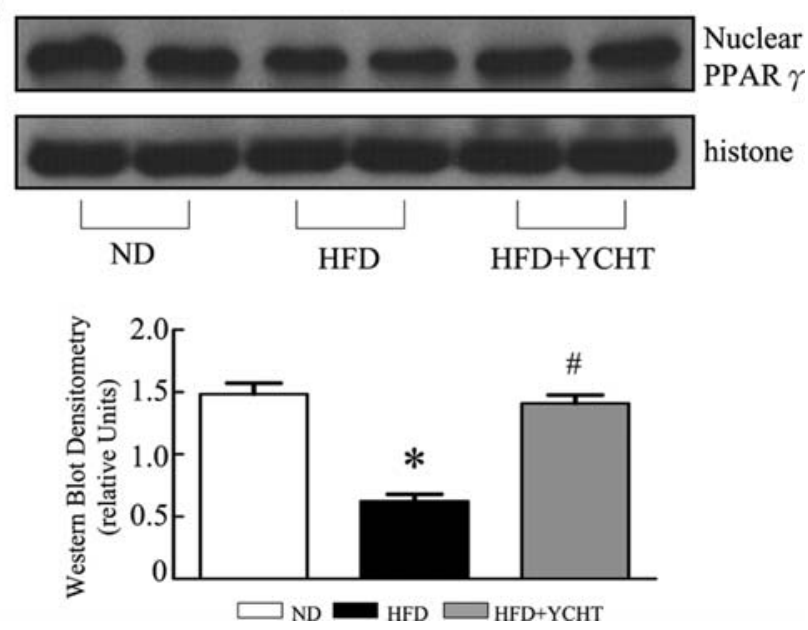
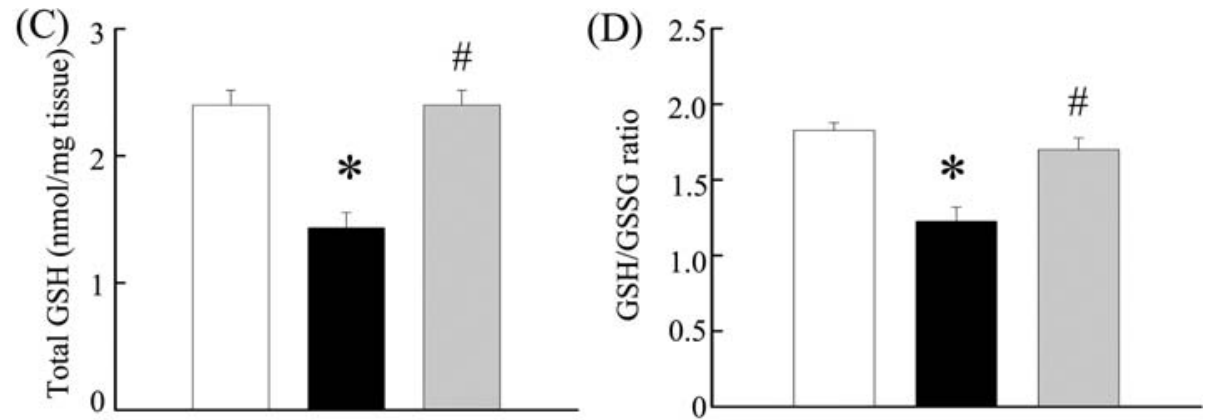

(E)

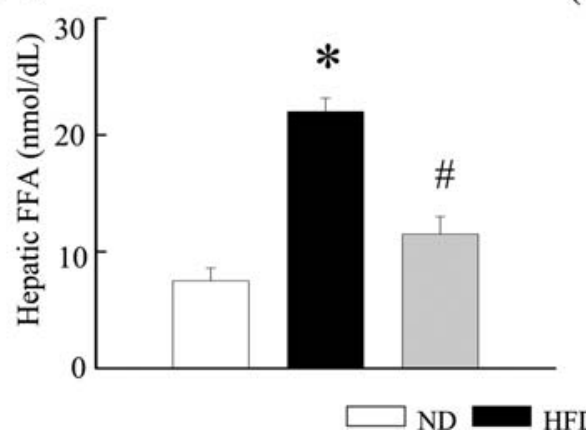

(F)

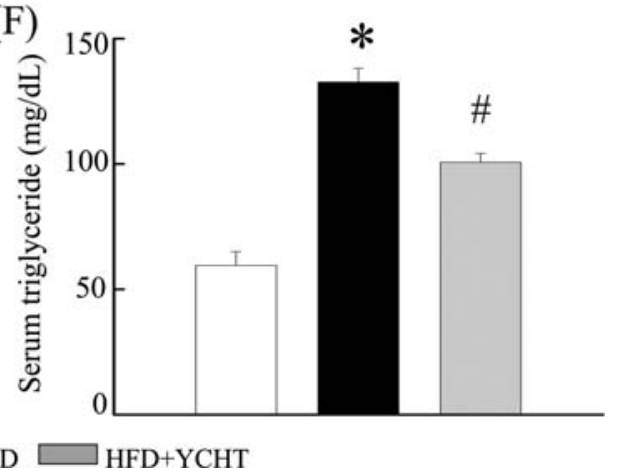

Figure 4. Amplification of hepatic TNF- $\alpha$, FAS, CPT-1 and PPAR $\gamma$ mRNA by RT-PCR from the livers of mice (A). Western blotting was performed to determine the effects of YCHT treatment on the activation of nuclear PPAR $\gamma(\mathrm{B})$. Each bar represents the mean value of experiments performed in triplicate. Hepatic total glutathione (GSH+GSSG) (C) and GSH/GSSG ratio (D) in the livers of obese mice and mice treated with YCHT. Free fatty acid quantification by ELISA (E). Serum triglycerides were determined by a spectrophotometry kit (F). The values are expressed as the mean \pm SEM ( $\mathrm{n}=5$ ). ${ }^{*} \mathrm{P}<0.05$ compared to the normal diet; $\mathrm{P}<0.05$ compared to the high fat diet

obese mice after YCHT treatment (Table I). Senescence marker protein (SMP30) was also identified by Western blotting (Fig. 5).

\section{Discussion}

PPAR is a transcription factor intimately connected to cell differentiation and the cellular metabolism of lipids, carbohydrates, and proteins. PPAR $\alpha / \gamma$-modulating drugs are used clinically. PPAR agonism increases tissue sensitivity to insulin action in diabetic patients and decreases hepatic triacylglycerol production by increasing fatty acid oxidation $(17,18)$. Both the previous results and those described here demonstrate that PPAR $\gamma$ is a key regulator of fatty acid oxidation. However, in response to elevated fatty acid levels, PPAR $\gamma$ activates expression of multiple components of the fatty acid synthesis pathway, resulting in an increased rate of oxidation. Obese mice exhibit increased hepatic expression of TNF- $\alpha$, a cytokine that is known to inhibit the propagation of insulin receptor initiated signals in hepatocytes $(19,20)$. Our results suggest a mechanism that could help to explain the association between increased hepatic TNF- $\alpha$ and obesityrelated fatty liver disease. Hepatic expression of FAS and CPT-1 are both increased in obese mice. Our results indicate that YCHT reduces hepatic TNF- $\alpha$ expression, enhances $\operatorname{PPAR} \gamma$ activity in obese liver nuclear extracts and downregulates FAS and CPT-1 mRNA. Thus, YCHT may at least partly reverse fatty liver disease, because it inhibits fatty acid 
Table I. Summary of expression data and identified hepatic proteins in Yin-Chen-Hao-Tang treated mice versus high fat diet mice.

\begin{tabular}{|c|c|c|c|c|c|}
\hline No. & $\begin{array}{l}\text { Accession } \\
\text { no. }\end{array}$ & Protein name & $\begin{array}{l}\text { ND vs. } \\
\text { HFD } \\
\text { (fold) }\end{array}$ & $\begin{array}{l}\text { HFD vs. } \\
\text { YCHT } \\
\text { (fold) }\end{array}$ & Function \\
\hline 1 & P20029 & $\begin{array}{l}\text { Glucose regulated } \\
\text { protein }(78 \mathrm{kDa})\end{array}$ & $>2.0$ & -2.0 & $\begin{array}{l}\text { Probably plays a role in facilitating the assembly of } \\
\text { multimeric protein complexes inside the ER, and mass } \\
\text { spectrometry. }\end{array}$ \\
\hline 2 & P11884 & Aldehyde dehydrogenase & $>2.0$ & -2.0 & An aldehyde $+\mathrm{NAD}^{+}+\mathrm{H}_{2} \mathrm{O}=$ an acid $+\mathrm{NADH}$ \\
\hline 3 & Q99LC5 & $\begin{array}{l}\text { Electron transferflavo- } \\
\text { protein }\end{array}$ & $>2.0$ & -2.0 & $\begin{array}{l}\text { The electron transfer flavoprotein serves as a specific } \\
\text { electron acceptor for several dehydrogenases, } \\
\text { including five acyl-CoA dehydrogenases, } \\
\text { glutaryl-CoA and sarcosine dehydrogenase. }\end{array}$ \\
\hline 4 & P38647 & Stress- 71 protein & $>2.0$ & -2.0 & $\begin{array}{l}\text { Implicated in the control of cell proliferation and } \\
\text { cellular aging. May also act as a chaperone. }\end{array}$ \\
\hline 5 & Q64374 & Regucalcin & $>2.0$ & -2.0 & $\begin{array}{l}\text { Calcium-binding protein which regulates } \mathrm{Ca}^{2+} \\
\text { signaling by regulating } \mathrm{Ca}^{2+} \text {-dependent enzymatic } \\
\text { activity in the liver and kidney. Decrease of } \mathrm{RGN} \text { leads } \\
\text { to the dysregulation of calcium signaling in the aged liver. }\end{array}$ \\
\hline 6 & P09103 & $\begin{array}{l}\text { Protein disulfide } \\
\text { isomerase }\end{array}$ & $>2.0$ & -2.0 & $\begin{array}{l}\text { Involved with other chaperones in the structural } \\
\text { modification of the TG precursor in hormone } \\
\text { biogenesis. Also acts a structural subunit of various } \\
\text { enzymes such as prolyl 4-hydroxylase and } \\
\text { microsomal triacylglycerol transfer protein MTTP. }\end{array}$ \\
\hline 7 & Q2PFL9 & Peroxiredoxin-6 & 2.0 & -2.0 & $\begin{array}{l}\text { Involved in redox regulation of the cell. Can reduce } \\
\mathrm{H}_{2} \mathrm{O}_{2} \text { and short chain organic, fatty acid, and } \\
\text { phospholipid hydroperoxides. May play a role in the } \\
\text { regulation of phospholipid turnover as well as in } \\
\text { protection against oxidative injury. }\end{array}$ \\
\hline 8 & P10649 & $\begin{array}{l}\text { Glutathione-S-transferase } \\
\text { Mu } 1\end{array}$ & 2.0 & -2.0 & $\begin{array}{l}\text { Conjugation of reduced glutathione to a wide number of } \\
\text { exogenous and endogenous hydrophobic electrophiles. }\end{array}$ \\
\hline 9 & P17563 & $\begin{array}{l}\text { Selenium binding } \\
\text { protein } 1\end{array}$ & 2.0 & -2.0 & $\begin{array}{l}\text { Selenium-binding protein which may be involved in } \\
\text { the sensing of reactive xenobiotics in the cytoplasm. } \\
\text { May be involved in intra-Golgi protein transport. }\end{array}$ \\
\hline 10 & Q9QXD7 & $\begin{array}{l}\text { Fructose-1,6-bis- } \\
\text { phosphatase } 2\end{array}$ & 2.0 & -2.0 & $\begin{array}{l}\text { D-fructose 1,6-bisphosphate }+\mathrm{H}_{2} \mathrm{O}=\mathrm{D} \text {-fructose } \\
\text { 7-phosphate }+ \text { phosphate. }\end{array}$ \\
\hline
\end{tabular}

synthesis in hepatocytes. This finding helps to explain how YCHT treatment reduces serum triglyceride concentrations. YCHT inhibitory effects on hepatic fatty acid synthesis might also contribute to the up-regulation of adiponectin in obese mice.

The 'Two-hit' hypothesis (21) that has been forwarded to explain hepatic steatosis consists of the initial insult, for example triglyceride or free fatty acid accumulation, followed by secondary insults that amplify hepatic production of reactive oxygen species (ROS) and induce cytokines, such as TNF- $\alpha$. Demonstrated for the first time in this study, YCHT has the ability to elicit hepatoprotective effects by enhanced tissue GSH expression and lower oxidative stress, which agrees with data showing increased SMP30 levels. High levels of SMP30 were maintained in the liver of young and adult rats. As the rate progresses to senescent stages, the amounts of SMP30 significantly decreased in the liver (22). Our results suggest that the down-regulation of SMP30 in the 


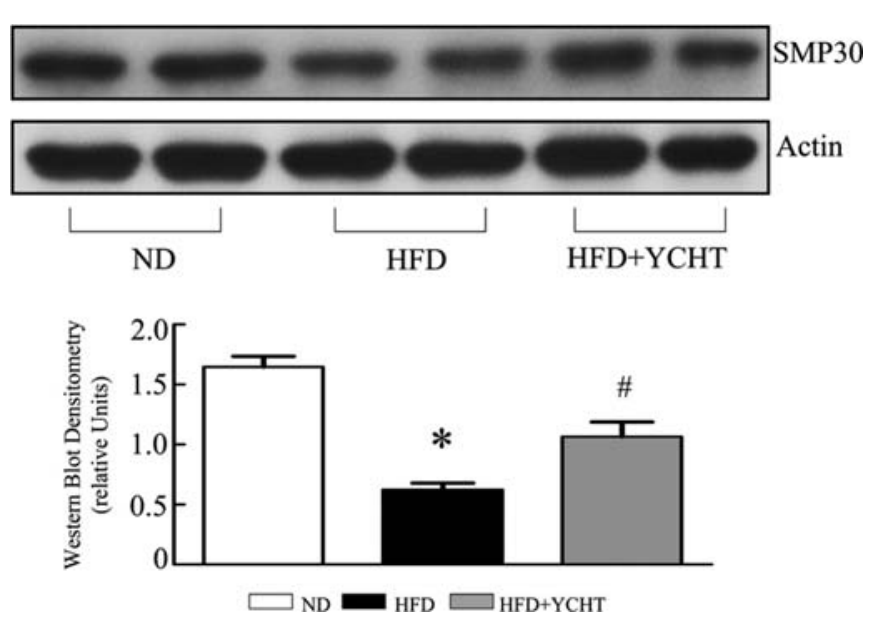

Figure 5. Partial 2D images of liver proteins from obese mice and animals receiving YCHT. The protein levels of SMP30 are shown by Western blotting. The blots are representative of three similar experiments. ${ }^{*} \mathrm{P}<0.05$ compared to the normal diet; ${ }^{\#} \mathrm{P}<0.05$ compared to the high fat diet.

liver may increase hepatic tissue susceptibility for harmful stimuli and may cause the deterioration of liver functions. The change in SMP30 expression after YCHT treatment may account for the attenuation of cellular functions by maintaining calcium homeostasis and ameliorate oxidative stress stimuli in obesity mice.

Proteomics approach identified a number of proteins whose expression levels were altered $>2$-fold in response to high fat feeding. These proteins fall into 4 different categories including: a) functions in drug metabolism such as selenium binding protein 1 (SBP1) and glutathione S-transferase (GST); b) proteins associated with oxidative stress, electron transfer flavoprotein, regucalcin (SMP30), peroxiredoxin 6 (PRDX6) and aldehyde dehydrogenase 2 (ALDH2), was down-regulated in high fat fed mice, and suggests an increase in reactive oxygen species and oxidative stress; c) molecular chaperones such as heat shock protein 60 and 70, whose functions are important for protein folding and anti-oxidation; d) enzymes in fructose catabolism such as fructose-1,6bisphosphatase 1 and glucose regulated protein 78 . These data provide insight into the molecular basis linking fat-induced metabolic shift to the development of metabolic syndrome characterized by hepatic steatosis and dyslipidemia.

The regulation of protein involved in ATP production such as ETF might be correlated with attenuation stress response after YCHT treatment in obese mice. Heat shock proteins play an important role in helping cells cope with a number of stresses such as heat shock and oxidative stress (23). YCHT increased production of heat shock proteins associated with better tolerance to obesity related metabolic stress, and therefore was not to deleterious to hepatocytes.

Fructose-1,6-bisphosphatase (FBPase) is a regulated enzyme in gluconeogenesis. Glucose-regulated protein 78 (GRP78) targets misfolded proteins for proteasome degradation, regulates calcium homeostasis, and serves as a sensor of endoplasmic reticulum stress. Therefore, it appears to play cytoprotective and anti-apoptotic functions. Interestingly, YCHT treatment shows a marked induction of GRP78 in livers of high-fat-fed hamsters. This effect is accompanied by induction of the anti-oxidant enzyme GST in livers in response to high fat feeding. Expressed abundantly in liver, PRX gene encodes a cytosolic peroxidase that functions to eliminate endogenous $\mathrm{H}_{2} \mathrm{O}_{2}$ generated from metabolism, which helps protect cells from oxidative stress and apoptosis (24). These results raise the possibility that $\mathrm{YCHT}$ exerts a protective effect on hepatic metabolism and liver function response to steatosis insults. The down-regulation of GST and ALDH2 in obese liver following oxidative stress has been confirmed in our model. Interestingly, YCHT serve as a compensatory mechanism to alleviate the oxidative damage caused by inappropriately increased fat deposition in liver. Under toxic condition (e.g. alcohol abuse) selenium levels of liver tissue decreased (25). Thus, SBP substitution in toxic-induced fibrosis may yield a therapeutic strategy targeted against SBP downregulation. Moreover, a rational explanation for the meaning of SBP1 regulation in obese mice may contribute to alter the progression of hepatic oxidative stress following YCHT treatment. Previous studies indicate that extracts contained in YCHT possess hypoglycemic, hypolipidemic activity $(26,27)$ and reduces hepatocyte apoptosis (28). These effects have been attributed to the anti-oxidant or other properties of the components of these extracts, but it is an interesting the therapeutic implication of YCHT on the management of obesity and steatosis may reduce liver injury and decrease the progression of fibrosis.

\section{Acknowledgements}

This work was supported by the National Science Council, Taipei, Taiwan, NSC96-2320-B-182-021 and CMRPD180241 supported by a Research Grant from the Chang Gung Memorial Hospital.

\section{References}

1. Grundy SM: Obesity, metabolic syndrome, and coronary atherosclerosis. Circulation 105: 2696-2698, 2002.

2. Eckel RH, Grundy SM and Zimmet PZ: The metabolic syndrome. Lancet 365: 1415-1428, 2005.

3. Mohanty P, Ghanim H, Hamouda W, Aljada A, Garg R and Dandona P: Both lipid and protein intakes stimulate increased generation of reactive oxygen species by polymorphonuclear leukocytes and mononuclear cells. Am J Clin Nutr 75: 767-772, 2002.

4. Angulo P: Nonalcoholic fatty liver disease. N Engl J Med 346: 1221-1231, 2002

5. Clark JM, Brancati FL and Diehl AM: Nonalcoholic fatty liver disease. Gastroenterology 122: 1649-1657, 2002.

6. Fruebis J, Tsao TS, Javorschi S, et al: Proteolytic cleavage product of 30-kDa adipocyte complement-related protein increases fatty acid oxidation in muscle and causes weight loss in mice. Proc Natl Acad Sci USA 98: 2005-2010, 2001.

7. Yamauchi T, Kamon J, Waki H, et al: The fat-derived hormone adiponectin reverses insulin resistance associated with both lipoatrophy and obesity. Nat Med 7: 941-946, 2001.

8. Oakes ND, Thalén PG, Jacinto SM and Ljung B: Thiazolidinediones increase plasma-adipose tissue FFA exchange capacity and enhance insulin-mediated control of systemic FFA availability. Diabetes 50: 1158-1165, 2001.

9. Mayerson AB, Hundal RS, Dufour S, et al: The effects of rosiglitazone on insulin sensitivity, lipolysis, and hepatic and skeletal muscle triglyceride content in patients with type 2 diabetes. Diabetes 51: 797-802, 2002.

10. Tiikkainen M, Hakkinen AM, Korsheninnikova E, Nyman T, Makimattila S and Yki-Jarvinen H: Effects of rosiglitazone and metformin on liver fat content, hepatic insulin resistance, insulin clearance, and gene expression in adipose tissue in patients with type 2 diabetes. Diabetes 53: 2169-2176, 2004. 
11. Inao M, Mochida S, Matsui A, et al: Japanese herbal medicine Inchin-ko-to as a therapeutic drug for liver fibrosis. J Hepatol 41: 584-591, 2004

12. Lee TY, Chang HH, Chen JH, Hsueh ML and Kuo JJ: Herb medicine Yin-Chen-Hao-Tang ameliorates hepatic fibrosis in bile duct ligation rats. J Ethnopharmacol 109: 318-324, 2007.

13. Yamamoto M, Ogawa K, Morita M, Fukuda K and Komatsu Y: The herbal medicine Inchin-ko-to inhibits liver cell apoptosis induced by transforming growth factor beta 1 . Hepatology 23 : 552-559, 1996

14. Lee TY, Chang HH, Wu MY and Lin HC: Yin-Chen-Hao-Tang ameliorates obstruction-induced hepatic apoptosis in rats. J Pharm Pharmacol 59: 583-590, 2007.

15. Lee TY, Chang HH, Kuo JJ and Shen JJ: Changes of hepatic proteome in bile duct ligated rats with hepatic fibrosis following treatment with Yin-Chen-Hao-Tang. Int J Mol Med 23: 477-484, 2009.

16. Reed DJ, Babson JR, Beatty PW, Brodie AE, Ellis WW and Potter DW: High-performance liquid chromatography analysis of nanomole levels of glutathione, glutathione disulfide, and related thiols and disulfides. Anal Biochem 106: 55-62, 1980.

17. Takahashi Y and Ide T: Effects of soy protein and isoflavone on hepatic fatty acid synthesis and oxidation and mRNA expression of uncoupling proteins and peroxisome proliferator-activated receptor gamma in adipose tissues of rats. J Nutr Biochem 19: 682-693, 2008.

18. Løvås K, Røst TH, Skorve J, et al: Tetradecylthioacetic acid attenuates dyslipidaemia in male patients with type 2 diabetes mellitus, possibly by dual PPAR-alpha/delta activation and increased mitochondrial fatty acid oxidation. Diabetes Obes Metab 11: 304-314, 2009

19. Solomon SS, Mishra SK, Cwik C, Rajanna B and Postlethwaite AE: Pioglitazone and metformin reverse insulin resistance induced by tumor necrosis factor-alpha in liver cells. Horm Metab Res 29: 379-382, 1997.
20. Uysal KT, Wiesbrock SM, Marino MW and Hotamisligil GS: Protection from obesity-induced insulin-resistance in mice lacking TNF- $\alpha$ function. Nature 389: 610-614, 1997.

21. Day CP and James OF: Steatohepatitis: a tale of two 'hits'? Gastroenterology 114: 842-845, 1998.

22. Fujita T: Senescence marker protein-30 (SMP30): structure and biological function. Biochem Biophys Res Commun 254: 1-4, 1999.

23. Nollen EA and Morimoto RI: Chaperoning signaling pathways: molecular chaperones as stress-sensing 'heat shock' proteins. J Cell Sci 115: 2809-2816, 2002.

24. Yang CS, Lee DS, Song CH, et al: Roles of peroxiredoxin II in the regulation of proinflammatory responses to LPS and protection against endotoxin-induced lethal shock. J Exp Med 204: 583-594, 2007.

25. Bergheim I, Parlesak A, Dierks C, Bode JC and Bode C: Nutritional deficiencies in German middle-class male alcohol consumers: relation to dietary intake and severity of liver disease. Eur J Clin Nutr 57: 431-438, 2003.

26. Wu SY, Wang GF, Liu ZQ, et al: Effect of geniposide, a hypoglycemic glucoside, on hepatic regulating enzymes in diabetic mice induced by a high-fat diet and streptozotocin. Acta Pharmacol Sin 30: 202-208, 2009.

27. Zhao XY, Qiao GF, Li BX, et al: Hypoglycaemic and hypolipidaemic effects of emodin and its effect on L-type calcium channels in dyslipidaemic-diabetic rats. Clin Exp Pharmacol Physiol 36: 29-34, 2009.

28. Lee TY, Chen FY, Chang HH and Lin HC: The effect of capillarisin on glycochenodeoxycholic acid-induced apoptosis and heme oxygenase- 1 in rat primary hepatocytes. Mol Cell Biochem 325: 53-59, 2009. 